\title{
Impactos da acupuntura no tratamento de pós-operatório do ligamento cruzado anterior em jogadores de futebol
}

Impacts of acupuncture on postoperative treatment of the anterior cruciate ligament in soccer players

Impactos de la acupuntura en el tratamiento posoperatorio del ligamento cruzado anterior en jugadores de fútbol

\section{Resumo}

O futebol é um esporte onde ocorrem muitas lesões, principalmente no joelho, e pelos mecanismos de movimento desse esporte o ligamento mais suscetível a essas lesões é o ligamento cruzado anterior. Com análise nesses dados o presente estudo tem por finalidade analisar a importância da acupuntura no tratamento de pós-operatório de ligamento cruzado anterior em jogadores de futebol, utilizando por método de pesquisa uma revisão da literatura por meio de pesquisa em bases de dados como PubMed, Scielo, Google Scholar, concluindo-se que a acupuntura auxilia muito o tratamento póscirúrgico principalmente no controle da dor e com efeitos muito melhores quando associada à fisioterapia, podendo acelerar a volta dos atletas às suas atividades esportivas. No entanto, pode-se ressaltar que mais estudos são necessários referente a esta temática.

Palavras-chave: Acupuntura; Futebol; Ligamento cruzado anterior; Pós-operatório; Esporte.

\begin{abstract}
Football is a sport where many injuries occur, mainly in the knee, and due to the movement mechanisms of this sport, the ligament most susceptible to these injuries is the anterior cruciate ligament. With the analysis of these data, the present study aims to analyze the importance of acupuncture in the postoperative treatment of anterior cruciate ligament in soccer players, using a literature review using research methods in databases such as PubMed, Scielo, Google Scholar, concluding that acupuncture helps a lot the post-surgical treatment, mainly in pain control and with much better effects when associated with physiotherapy, being able to accelerate the athletes' return to their sports activities. However, it can be emphasized that more studies are needed regarding this theme.
\end{abstract}

Keywords: Acupuncture; Soccer; Anterior cruciate ligament; Postoperative; Sport.

\section{Resumen}

El fútbol es un deporte donde se producen muchas lesiones, principalmente en la rodilla, y debido a los mecanismos de movimiento de este deporte, el ligamento más susceptible a estas lesiones es el ligamento cruzado anterior. Con el análisis de estos datos, el presente estudio tiene como objetivo analizar la importancia de la acupuntura en el tratamiento del ligamento cruzado anterior posoperatorio en futbolistas, mediante una revisión de la literatura mediante la investigación en bases de datos como PubMed, Scielo, Google Scholar, concluyendo que la acupuntura ayuda mucho al tratamiento posquirúrgico, principalmente en el control del dolor y con efectos mucho mejores cuando se asocia a la fisioterapia, pudiendo acelerar el regreso de los deportistas a sus actividades deportivas. Sin embargo, se puede enfatizar que se necesitan más estudios sobre este tema.

Palabras clave: Acupuntura; Fútbol; Ligamento cruzado anterior; Postoperatorio; Deporte.

\section{Introdução}

A cada ano que se passa, com o advento da modernidade, novas tecnologias surgem, incluindo na área da saúde, onde se observa a criação de novas técnicas, procedimentos, medicações, etc. E com essa nova modernidade, foi deixado de lado alguns tratamentos integrativos como a acupuntura, que é uma técnica milenar com muitos recursos e benefícios para a saúde. 
No cenário desportivo não é diferente, a acupuntura ainda é pouco utilizada como tratamento complementar, e principalmente no tratamento de pós-operatório dos atletas. Este estudo visa analisar a utilidade da acupuntura como uma forma de tratamento complementar para o tratamento de pós-operatório de ligamento cruzado anterior (LCA) em jogadores de futebol com o objetivo de recuperar os atletas.

Além de todos os benefícios que a acupuntura pode trazer para a saúde dos atletas, é uma terapia alternativa e complementar de baixíssimo custo, podendo ser utilizada por instituições desportivas de grande ou pequeno porte, pois independe de se ter muitos ou poucos recursos nessas instituições.

\section{Metodologia}

O presente estudo consiste em uma revisão integrativa que entre as revisões é a mais ampla, permitindo acrescentar estudos experimentais ou não-experimentais, dados da literatura empírica ou teórica, assim como a inclusão de muitos propósitos (definição de conceitos, revisão de teorias e evidências, análise de problemas metodológicos (Souza, Silva \& Carvalho, 2010).

Os resultados serão apresentados sobre forma qualitativa, que segundo Richardson (2018), permite descrever a complexidade de determinado problema, analisar a interação de certas variáveis, compreender e classificar processos dinâmicos vividos por grupos sociais, por meio de coletas de informações de fontes primárias e secundárias, incluindo livros, revisão bibliográfica e estudos.

A coleta de dados se deu através de pesquisa em bases de dados eletrônicas como PubMed, Scielo, Google Scholar, utilizando os descritores "pós-operatório AND acupuntura", "pós-operatório ligamento cruzado anterior", "acupuntura AND esporte", "pós-operatório jogadores de futebol", "acupuntura AND jogadores de futebol”. Como critério de inclusão foram selecionados trabalhos publicados na íntegra, nos idiomas português, inglês e espanhol. Foram excluídos os editoriais, trabalhos de conclusão de curso, cartas ao editor e relatos de experiência. E então, foi realizada leituras dos títulos e resumos, seguida da leitura na íntegra, sendo excluídos aqueles não pertinentes à temática e com duplicidade. Desta forma, dos 117 artigos encontrados, sendo 34 PubMed, 17 Scielo e 66 Google Scholar, havendo seleção final de 61 trabalhos. O fluxograma abaixo sintetiza as etapas metodológicas do trabalho.

Figura 1. Fluxograma de coleta de dados.

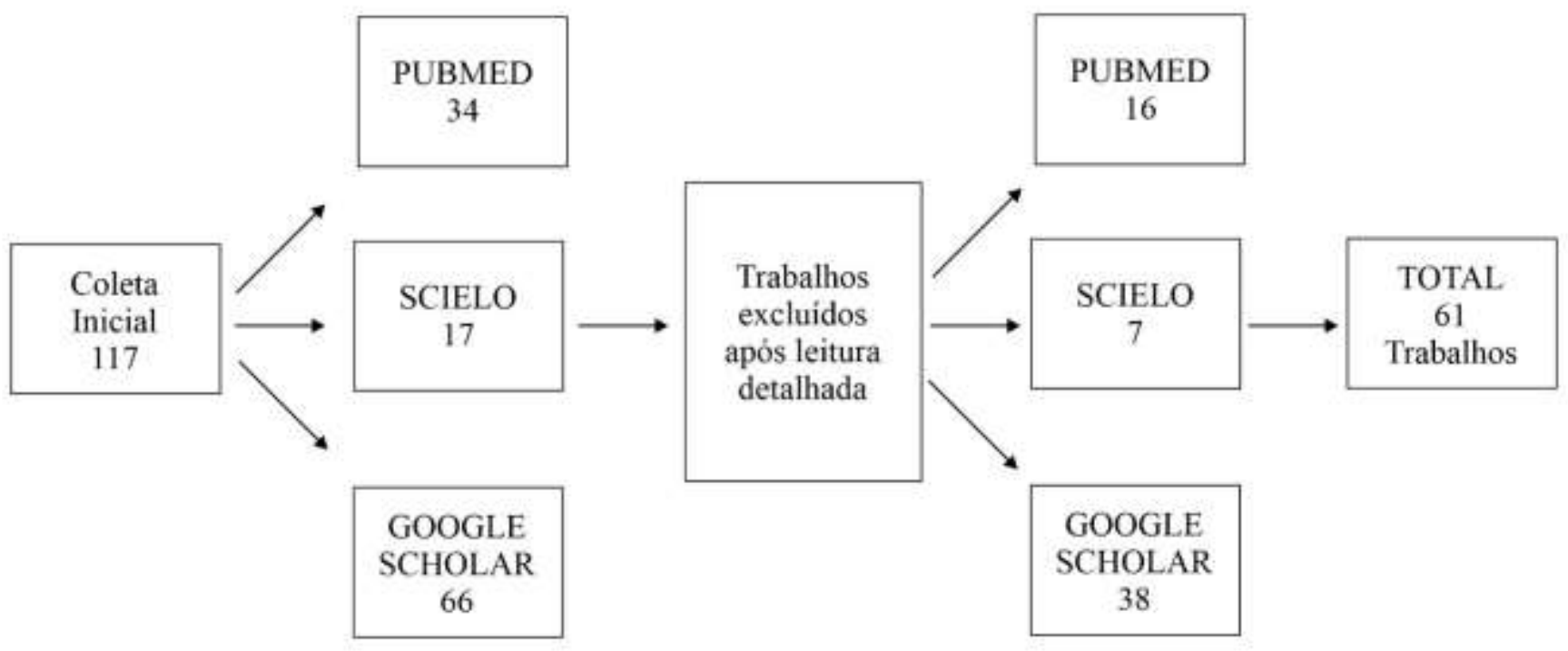

Fonte: Autor. 


\section{Resultados e Discussão}

Uma lesão no futebol é definida como qualquer tipo de ocorrência sofrida por um jogador em competição ou em treino que o obrigue a interromper suas atividades em treino ou em jogo, tendo sido estimado que três em cada quatro jogadores de futebol de elite sofram, por ano, uma lesão limitadora da sua performance (Hagglund et al., 2005). Segundo Astur (2016), quando avaliou a incidência de lesões no futebol, na corrida e na musculação, o esporte mais lesivo foi o futebol, em segundo lugar foi a corrida nas lesões ligamentares e meniscais isoladas e musculação nas lesões associadas. E apesar de a lesão do joelho não ser a que apresenta maior incidência, entre as lesões graves é a mais comum entre os jogadores de futebol (Hagglund et al., 2005). O LCA é enfatizado como um dos ligamentos do joelho mais lesionados frequentemente; e pela elevada incidência e severidade, a lesão do LCA continua a ser a lesão mais temida entre os jogadores de futebol (Boone, 2006).

Portanto, de acordo com os dados acima citados, Soares (2007) relata que as lesões de LCA são frequentemente responsáveis pelo abandono precoce da prática desportiva e surgimento de possíveis complicações na integridade e funcionalidade da articulação do joelho dos jogadores de futebol, dada a incidência, severidade, custos e tempo de interrupção da atividade durante o período da lesão. E, como dito anteriormente, por manter muitos atletas afastados das competições durante, pelo menos, quatro meses da época competitiva, a lesão do LCA é um acontecimento grave que requer tratamento cirúrgico (Roi et al., 2006).

Em relação aos fatores que levam à lesão, Hughes e Watkins (2006) atestam que existem duas grandes categorias de lesão no futebol, sendo elas, lesões de contato ou acidentais que ocorrem pela pressão exercida em um momento ou ação excedendo os limites de resistência do tecido; e lesões de não-contato ou sobreuso originadas pelo estresse repetido no tempo. Porém é difícil estabelecer os estudos relativos aos fatores de risco de lesão pela complexa interação entre os diversos fatores e dificuldade de englobar todos os potenciais fatores de risco no mesmo estudo (Söderman et al., 2001). Para Soares (2007), é consensual que à medida que se tem um aumento do nível competitivo aumenta também o risco de lesão, sendo a taxa de lesões em competição claramente superior e sabendo-se que o mecanismo mais frequente de lesão do LCA se relaciona com movimentos de rotação, movimentos que são dificultados pela fixação das travas das chuteiras ao solo, portanto, o tamanho (tipo) das travas pode constituir um fator de risco, uma vez que ao não permitir a rotação fluida do pé no solo, pode provocar a lesão dos ligamentos do joelho.

A lesão do LCA ocorre pela ação de uma carga excessiva sobre o ligamento, como resultado de um movimento anormal da articulação tíbio-femoral levando à falha dos suportes mecânicos que a estabilizam (HUGHES E WATKINS, 2006). Hertel, Dorfman e Braham (2004), acreditam que alguns desalinhamentos dos membros inferiores têm sido associados ao aumento do risco de lesão do LCA, pelo aumento da tensão exercida sobre o ligamento, destacam os desalinhamentos do pé e pelve que parecem contribuir para o maior risco de lesão do LCA, principalmente se caso haja aumento da depressão do navicular e a anteroversão da bacia.

Como dito anteriormente, os fatores de risco são muitos e difíceis de serem todos relatados, portanto, analisando os mecanismos de lesão, Palastanga, Field e Soames (2000) descrevem que o LCA se fixa ligeiramente com o corno anterior do menisco lateral e fixa-se posterior, lateral e proximalmente na parte posterior da superfície medial do côndilo femoral lateral. Noronha (2006), aponta que o LCA tem a função de estabilizar a articulação em todos os movimentos de rotação e translação, sendo que a principal função é prevenir a translação anterior da tíbia em relação ao fêmur; ele também evita a rotação interna e externa da tíbia em relação ao fêmur, principalmente na extensão do joelho e limita a deformação em varo e valgo quando em extensão.

Seguindo essa ideia, a maioria das lesões de LCA ocorre com flexão do joelho entre $0^{\circ}$ e $30^{\circ}$, normalmente em situações de não-contato, em corrida ou em salto (BODEN et al., 2000). Segundo Noronha (2006), gerando grande tensão sobre o 
ligamento através de leve flexão, varo e rotação externa; e com menor frequência pode haver a ruptura do LCA quando o joelho está em flexão, valgo e com rotação interna do fêmur.

Sendo uma dessas situações de lesão a corrida, os dentre os movimentos associados à lesão estão a mudança de direção, rápida desaceleração, frenagem espontânea e movimentos de torção; enquanto no salto, os movimentos mais comuns são a recepção no solo em varo e rotação interna ou em valgo e rotação externa (Cerulli et al., 2003).

Acrescentando às informações anteriores, para Noronha (2006), a ruptura do LCA pode ocorrer também em hiperextensão, por atrito com chanfradura intercondiliana, quando apresenta dimensão inferior a normal, e também descreve que recurvatum pode promover a ruptura do ligamento, principalmente se estiver associada à contratura do quadríceps, gerando uma subluxação anterior da tíbia, o que leva aos jogadores a relatarem que o joelho "saiu do lugar" e ter sentido algo "estourar" dentro do joelho, correspondendo ao ultrapassar o limiar da resistência das fibras do LCA; e poucos minutos após a lesão, surge um edema mais ou menos volumoso.

Quando se tem uma ruptura total, o possível tratamento é cirúrgico e de acordo com Arliani et al. (2012), a queixa de instabilidade e falseio do paciente, além da manobra de pivot-shift positiva no exame físico são os fatores considerados, na decisão de operar o paciente, mais determinantes. Para Lima et al. (2018), as principais indicações cirúrgicas descritas para a reconstrução do LCA, se combinada com tenodese extrarticular lateral ou reconstrução do ligamento anterolateral (LAL) são a revisão do LCA, exame físico com pivot-shift grau 2 ou 3, prática de esporte com mecanismo de pivot e/ou de alto nível, frouxidão ligamentar e fratura de Segond. De acordo com Frobell et al. (2010), a reconstrução cirúrgica é o tratamento padrão em atletas, sendo realizadas em média 200.000 reconstruções do LCA anualmente nos Estados Unidos e custos estimados em 3 bilhões de dólares.

Levando em consideração todas essas variáveis e o consenso que o LCA não cicatriza adequadamente após a lesão, como procedimento padrão, existem inúmeras técnicas cirúrgicas de reconstrução para recuperar a estabilidade, em estudos realizados por Arliani et al. (2012), foi constatado que os cirurgiões de joelho brasileiros utilizam, em sua maioria (82,3\%), os tendões flexores grácil e semitendíneo e o terço central do tendão patelar ipsilateral à lesão em 53,5\%.

Segundo Francis, Thomas e McGregor (2001), os cirurgiões do Reino Unido, neste mesmo ano do estudo, também relataram preferência por estes tipos de enxerto. Enquanto nos estudos de Heijine e Werner (2010), apontaram que a reconstrução com tendão patelar quando comparada com tendão de flexores levou a joelhos mais estáveis, com menor frouxidão anterior do joelho e menos instabilidade rotacional. Barker et al. (2010), relatam que o os enxertos de isquiotibiais têm maior incidência de infecção do que os auto e aloenxertos de tendão patelar. Sun et al. (2009), observaram que também podem ser utilizados enxertos com o terço distal do quadríceps e aloenxertos, por mostrarem bons resultados quando utilizados nas reconstruções de LCA.

Nessas condições, Rourgraff et al. (1993), observaram, em estudo animal, que o neoligamento passava por um processo de necrose avascular atingindo a fase de ligamentização aos seis meses estando, portanto, mais suscetível a rupturas durante esta fase. Baseado nesses dados, Thiele et al. (2009), observaram que os protocolos de reabilitação eram muito conservadores em relação ao tempo de recuperação do movimento de extensão do joelho, exercícios com carga, apoio e liberação para as atividades esportivas. No entanto, Rourgraff et al. (1993), em outro estudo, desta vez uma análise histológica do neoligamento em humanos, demonstraram que, ao contrário dos estudos em animais, o enxerto em humanos não apresenta estágio de necrose completa; a porção central do enxerto apresenta necrose, mas a porção superficial encontra-se totalmente viável, sendo observado pela proliferação celular e neovascularização proeminente, permitindo que o enxerto está parcialmente viável a partir da terceira semana do pós-operatório.

No pós-operatório de reconstrução de LCA, pode haver alterações sensoriais devidas às lesões do ramo infrapatelar do nervo safeno durante a retirada do enxerto de tendões flexores, podendo cometer até $74 \%$ dos pacientes dependendo do tipo de incisão utilizada na coleta do enxerto, podendo alterar essa frequência de lesões (Sanders et al., 2007). 
Algumas variáveis podem ou não interferir na recuperação do jogador no pós-operatório da reconstrução de LCA, como o uso de brace no período do pós-operatório, em um estudo realizado no Reino Unido foi observado que somente $30 \%$ dos ortopedistas imobilizam os seus pacientes no pós-operatório (Francis, Thomas \& Mcgregor, 2001). Como também foi encontrado pela literatura mais recente em estudo realizado por Birmingham et al. (2008) que também não sugere o uso de brace funcional após a reconstrução do LCA. E quanto aos médicos brasileiros, a maioria deles (71,7\%), não utilizam o brace (Arliani et al., 2012).

Além do brace, ainda pode ser observado a opção da utilização ou não de infiltração intra-articular de anestésico, que no ponto de vista de Musil, Sadovsky e Stehlik (2007), o uso de anestésico intra-articular no pós-operatório tem efeito significativo na diminuição no uso de medicação pelo paciente durante um período de 24 horas.

Sendo baseado por todos esses dados, pode-se dizer que o tratamento pós-operatório é de suma importância na recuperação dos jogadores de futebol, segundo Soares (2007), nesse processo de reabilitação pós-cirúrgico é fundamental que decorra tempo suficiente para a cicatrização total dos tecidos, ainda que o jogador pareça estar totalmente recuperado do ponto de vista funcional e mecânico, retornando gradualmente à competição com atenção maior a sinais e sintomas de piora ou recidiva da lesão. E em estudo mais recente Arliani et al. (2019) relatam que os jogadores são liberados para retorno ao esporte sem restrições após 6 a 8 meses após cirurgia e o parâmetro usado para liberação e retorno ao esporte é o teste de força isocinético.

Por ter tanta importância, a reabilitação deve ser muito bem realizada, levando em consideração alguns fatores: a retirada do terço médio do tendão patelar, a intervenção cirúrgica, presença de dor, entre outros podem levar à atrofia muscular no pósoperatório, principalmente no grupo extensor do joelho (Shelbourne \& Patel, 1996); a força do quadríceps está associada significativamente à estabilidade funcional do joelho, antes e após a operação (Keays et al., 2003). Portanto, segundo Tyler et al. (2004), tem sido de grande preocupação no pós-operatório a recuperação da condição muscular.

Existem muitos protocolos de reabilitação de pós-operatório de reconstrução de LCA, e por muito tempo foi utilizado protocolos mais conservadores, mas hoje em dia existem protocolos de reabilitação mais acelerados (Ramos et al., 2019). Foi realizado por Shelbourne e Nitz (1990) um estudo onde compararam dois tipos de protocolos de reabilitação, um mais conservador e outro acelerado, os pacientes submetidos ao protocolo acelerado teve uma recuperação da extensão do joelho mais acelerada, havendo menos casos em que a perda de extensão levava a uma nova intervenção cirúrgica; exercícios em cadeia cinética fechada também ajudaram a reduzir dores anteriores no joelho, aumentar a estabilidade subjetiva e recuperar força de quadríceps mais rapidamente. Em um outro estudo, Shelbourne e Gray (1997), constataram que a mobilização e fortalecimento precoce no pós-operatório imediato proporcionaram resultados positivos.

Em estudo realizado por Thiele et al., (2009), foi observado, em uma reabilitação com protocolo acelerado, a recuperação do trabalho total foi em média de $94,2 \%$ nos músculos flexores e de $74,1 \%$ nos músculos extensores, em quatro meses de pós-operatório. E Shelbourne et al., (1991), em estudo realizado com grupos seguindo a reabilitação com protocolo acelerado e outro com protocolo tradicional, observou recuperação da performance da musculatura extensora de 70\% após 13 semanas após o procedimento cirúrgico. Os resultados obtidos nesses estudos, sugerem que o protocolo acelerado pode acelerar a reabilitação no pós-operatório de reconstrução de LCA. E como opção de tratamento complementar aliada a fisioterapia, pode ser incluído no protocolo de reabilitação o uso de várias técnicas, dentre elas a acupuntura.

\section{Acupuntura}

A acupuntura é um dos métodos mais antigos de tratamento no mundo. Esta técnica surgiu na China há aproximadamente 4.500 anos, começou com a utilização de pedras pontiagudas (pedra bian, em chinês) para puncionar ou pressionar a pele, sendo substituída depois por um osso pontiagudo e com a descoberta dos metais passaram a ser utilizadas 
agulhas de ouro e prata (Jayasuriya, 1995). No Brasil a acupuntura chegou com imigrantes chineses no século XIX (Rio de Janeiro) e com os japoneses no século XX (São Paulo). Despertou-se o interesse pela acupuntura na década de 1960 através do movimento hippie e na década de 1970, com uma maior divulgação pela mídia da cultura chinesa no Brasil enfocando a Medicina Tradicional Chinesa (MTC). Estudantes de medicina começaram a se interessar e organizar cursos informativos referente a essa nova técnica em um processo gradual e progressivo do assunto no meio acadêmico (Siqueira \& Viegas, 2019).

A acupuntura é uma técnica da MTC utilizada para a manipulação do $Q i$ através dos canais de energia, o que permite promover a harmonia do corpo e da mente (Siqueira \& Viegas, 2019).

A MTC segue as teorias do yin e yang e dos cinco elementos, que tem por conceito que o campo eletromagnético da vida ou $Q i$ no organismo flui por todos os órgãos e vísceras (Zang $F u$ ) e esta comunicação ocorre pelos meridianos, portanto, alterações nesse fluxo causariam sintomas de acúmulo (ou Yang) ou deficiência (ou Yin) de energia (Machado, 2011).

Contatorer, Tesser e Barros (2018) afirmam que o conjunto desses sinais é codificado por parâmetros extremamente subjetivos para a racionalidade médica ocidental porque representam alterações no fluxo do $Q i$, do yin (hipofuncionamento) e do yang (hiperfuncionamento), próprias do organismo de cada indivíduo e o tratamento visa redirecionar o fluxo do $Q i$ tonificando a deficiência, sedando o excesso ou dispersando a estagnação. O princípio terapêutico visa remover os fatores patológicos, restabelecendo o equilíbrio e ativar a livre circulação de $Q i$ e Xиe (sangue) (Maciocia, 2007).

A MTC utiliza métodos específicos de diagnóstico e o uso da acupuntura também é baseada nesses métodos. Existem quatro aspectos na semiologia da MTC para o diagnóstico da doença, denominados de Quatro Métodos Diagnósticos ou Si Zhen. Eles incluem a inspeção, audição e olfação, anamnese, palpação de pulso e palpação de estruturas corporais (WEN, 2011). Como o corpo humano é um conjunto integrado, as alterações patológicas em determinados locais podem afetar o corpo como um todo e uma alteração em um órgão interno pode se apresentar nos cinco órgãos dos sentidos, extremidades e superfície corporal (WEN, 2011).

Em relação à dor e disfunção musculoesquelética se distingue as categorias de parâmetros semiológicos para o seu diagnóstico, avaliando-se aspectos sensoriais, temporais, fatores de agravamento e melhora, topografia e processos fisiopatológicos divididos em superfície e profundidade e definindo o padrão de patogenia associado ao quadro de dor (Capili, Anastasi \& Geiger, 2010).

As agulhas, quando inseridas, são giradas, aquecidas ou podem ser estimuladas com correntes elétricas de baixa intensidade, ultrassom ou luz de determinados comprimentos de ondas (Lee et al., 1975). Os locais onde são inseridas as agulhas são chamados de acupontos, que é uma região da pele com grande concentração de terminações nervosas e tem relação íntima com nervos, vasos sanguíneos, tendões, periósteos e cápsulas articulares (Medina, 2011) e a localização destes deve ser o mais precisa possível para o sucesso do tratamento. Esses pontos, quando estimulados, permitem a ativação ou sedação da energia que circula ao longo dos meridianos (Siqueira \& Viegas, 2019).

Como dito anteriormente, os acupontos podem se localizar ao longo de um meridiano ou não. Com grande importância, a literatura nos apresenta a escolha de pontos e meridianos a serem trabalhados a respeito de cada patologia (Medina, 2011). Cada ponto tem seus efeitos e indicações específicas, diferentes entre si, porém, os pontos de um mesmo meridiano apresentam efeitos terapêuticos muito semelhantes e de acordo com eles, podemos dividir esses pontos em três categorias quanto a seu efeito, a saber, efeitos sistêmicos, locais e à distância (Wen, 2011). Eles apresentam funções terapêuticas além das propriedades atualmente associadas aos pontos-gatilho miofasciais (PGM), sua utilização é indicada não só para dores musculoesqueléticas quanto também para normalizar a funcionalidade do organismo (Capili, Anastasi E Geiger, 2010).

Os meridianos são qualificados como canais que transportam o sangue e energia vital ( $Q i)$ e não passam propriamente pela pele, mas em camadas com profundidades diferentes da superfície do corpo. Esses canais de energia também estão 
relacionados com as áreas de influência dos órgãos no corpo, sendo importante na escolha de pontos de acupuntura para o tratamento de distúrbios localizados (Medina, 2011).

A acupuntura é reconhecida pela Organização Mundial da Saúde (OMS) como tratamento principal e/ou complementar para as várias patologias distintas, entre elas: alergias, enxaquecas, problemas gastrointestinais, algias variadas e, de acordo com Santos et al. (2009), vários estudos demonstram a profunda influência da acupuntura sobre os problemas físicos e emocionais, tendo a recomendação da associação desta técnica com outros tipos de tratamento, incluindo a fisioterapia. Além de ser uma técnica de baixo custo, não tóxica e de poucos efeitos colaterais, portanto utilizada em vários países atualmente (Moniz, 2015).

Inclusive, no meio esportivo, a acupuntura vem sendo mais utilizada por apresentar resultados imediatos e não ser considerado doping. Além dos seus efeitos demonstrarem resultados positivos quanto testada na melhora de performance aeróbica, força, desempenho, flexibilidade, bem-estar físico e mental dos atletas, com a sua utilização também se evita o uso de medicação e melhora a prática da atividade esportiva, promovendo analgesia em casos de dor gerada pelo treinamento (Frasca, 2011). Luna e Fernandes Filho (2005) relatam que determinados pontos de acupuntura quando estimulados podem melhorar a performance esportiva, plasticidade muscular e qualidade física, assim prevenindo lesões. E Yang, Liu e Gao (2006) afirmam que a acupuntura proporciona uma melhora da velocidade dos atletas por melhorar significativamente as condições biomecânicas de força.

Essa técnica também pode ser utilizada, além de tonificar e relaxar a musculatura dos atletas, como prevenção do equilíbrio de energias. A acupuntura causa efeito analgésico, mas também estimula outros efeitos biológicos, dentre eles a ativação do hipotálamo-hipófise, promovendo efeito sistêmico com o aumento de neurotransmissores e neuro-hormônios e pode promover também excitabilidade de nervos periféricos com a regeneração de fibras nervosas, melhora da nutrição tecidual e consequente melhora energética da musculatura (Santos, Kawano \& Banja, 2008). Segundo Ohnishi (2007), esse efeito de bemestar físico, que a acupuntura promove, é o responsável por aumentar e melhorar a performance do atleta em sua respectiva modalidade, sendo controlado por 3 sistemas: endócrino, imune e nervoso que são influenciados por treinamentos de alta intensidade e volume. Desta forma, a acupuntura pode gerar alívio de tensão da musculatura, aumento do fluxo sanguíneo local, limiar de dor e melhora da modulação do sistema nervoso autônomo que produz substâncias antioxidantes e promovendo a diminuição do estresse oxidativo.

Em um estudo de Dias Junior e Marino (2019), avaliando jogadores de futebol, em 100\% dos atletas testados o tratamento com acupuntura proporcionou um efeito positivo em prevenção de lesão muscular em relação ao grupo controle e aos grupos que foram utilizados apenas um ponto específico e auricular, sendo que Grupo 1 foi tratado com pontos de Baço-Pâncreas (BP) e Estômago (E) com objetivo de tonificar elemento terra e melhorar energeticamente a musculatura, Grupo 2 - de acordo com o desequilíbrio energético do pentagrama e em conjunto com a auriculoterapia, Grupo 3 - ponto E36, Grupo 4 - ponto BP6, Grupo 5 - auriculoterapia e Grupo Controle. Concluindo-se que os Grupos 1 e 2 não obtiveram lesões, no Grupo Controle obtiveram 6 lesões, os Grupos 3 e 5 obtiveram uma lesão e o Grupo 4 obtiveram 2 lesões. E analisaram que além de apresentar os resultados previstos em relação à prevenção de lesões musculares, a acupuntura pode ser utilizada na melhora da performance e funcionalidade dos atletas com o objetivo de atingir melhores resultados dentro de seus respectivos esportes, por ser uma técnica de baixo custo e não estar envolvida a uso de medicações ou substâncias ilícitas.

\section{Acupuntura no Pós-Operatório de LCA}

A acupuntura pode contribuir na potencialização do processo de reabilitação do aparelho locomotor agindo direta ou indiretamente nas valências físicas quanto ao sistema musculoesquelético, melhorando a performance dos atletas em sua respectiva atividade esportiva, acelerando a recuperação diminuindo o tempo de ausência e suas atividades físicas (Tansini et al., 2015). Esta técnica também tem sido indicada para o tratamento de casos de dor pelo estímulo da liberação de substâncias 
analgésicas e anti-inflamatórias (Van de Berg et al., 2010) e tem eficácia comprovada em diversas situações como relatam Sertel et al. (2009).

Em estudo realizado por Min (2004) se observa que a acupuntura promove uma reação fisiológica associada à liberação de substâncias em várias áreas cerebrais, como beta-endorfina, que junto com os outros dois opioides (encefalina e dinorfina) são partes integrais do mecanismo natural de supressão de dor. Com a liberação dessas substâncias é modulada a passagem da mensagem dolorosa e consequentemente é produzido um potente efeito analgésico.

Para Santos et al. (2009), a acupuntura age não só na medula espinhal pela modulação dos estímulos nociceptivos por inibição pré-sináptica devido à liberação de encefalinas e dorfinas, mas também, age a nível mesencéfalico, através da secreção de encefalinas e a ativação do sistema inibitório descendente resultando na liberação de serotonina e norepinefrina.

A dor é uma das manifestações clínicas mais frequentes e pode, segundo a MTC, ser classificada em 3 tipos distintos na dependência dos fatores energéticos causais: Fator Yang - polaridade positiva, dor do tipo pontada/facada/agulhada, dor aguda, superficial, violenta, transfixante, que melhora com o frio, repouso, imobilização e piora com o calor, movimentos musculares, exercícios, pressão. compressão; Fator Yin - aumenta a polaridade negativa, dor contínua, sentida mais internamente (dor profunda), graus variáveis de intensidade, crônica, sensação de peso e adormecimento, melhora com o calor, movimentos musculares, exercícios, pressão, compressão e piora com o frio, repouso e mudança de tempo; Fator Falso-Yang - aumenta a polaridade positiva, dor intermediária entre o fator yin e o fator yang (Saidah, 2001).

Para Capili, Anastasi e Geiger (2010), a dor pode também ser classificada por critérios qualitativos (variações de acordo com fator patogênico) e quantitativos (deficiência ou excesso / $Q i$ ortodoxo versus fator patogênico). No caso de excesso se deve diferenciar entre estase de sangue ou estagnação de $Q i$, se deve identificar as causas desse excesso (topográficos: processo doloroso relacionado aos órgãos internos/Zang $F u$; ou sistema musculoesquelético: Jing Luo) e em relação aos fatores patogênicos.

Em relação à concepção da MTC, de acordo com Yamamura (2009), o joelho está associado a energia do Fígado (Gan) e com os 6 canais de energia que passam por esta região e são responsáveis por atividades que envolvem a articulação do joelho, nervos e circulação sanguínea, e também pelo processo de adoecimento não-traumático ou pós-traumático do joelho. Esses 6 canais são divididos em: 3 canais de energia Yang que são constituídos pelos canais de energia do Estômago (Wei), Vesícula Biliar (Dan) e Bexiga (Pangguang), e 3 canais de energia Yin do pé que são os canais de energia do Fígado (Gan), Rins (Shen) e Baço-Pâncreas (Pi) (Saidah et al., 2003).

Yamamura (1993) associou as estruturas do joelho com esses canais de energia: o canal do Estômago (Wei) se encontra na parte anterolateral do joelho e está associado à membrana sinovial, patela, tendão do quadríceps, tendão patelar, plexo nervoso peripatelar e partes moles da região anterior do joelho; o canal da Vesícula Biliar (Dan) se encontra na face lateral do joelho e está associado aos côndilos laterais do fêmur e tíbia, menisco lateral, ligamento colateral lateral (LCL), tendão do bíceps femoral e nervos fibulares; o canal da Bexiga (Pangguang) se encontra na fossa poplítea e está associado as estruturas moles desta região e nervo tibial; já os canais do Fígado (Gan), Rins (Shen) e Baço-Pâncreas (Pi) se encontram na face medial do joelho e estão associados ao côndilo medial do fêmur e tíbia, menisco medial, ligamento colateral medial (LCM), cápsula articular medial, tendões do semitendíneo, grácil e semimembranoso e com o nervo safeno.

Partindo desses princípios Saidah (1997) relata que inserindo as agulhas nos pontos situados no joelho sadio, através de um arco reflexo sômato-somático contralateral pode interferir em mecanismos que geram desequilíbrio do joelho afetado e consequentemente também na dor e em seus movimentos. Em estudo realizado por Saidah et al. (2003), foi constatado a eficácia da técnica "ao aposto", que pode ser utilizada em situações em que é difícil a inserção da agulha de acupuntura no joelho lesionado, como em uso de aparelho gessado, pós-fratura, pós-cirurgia, processos inflamatórios e infecciosos graves, mas principalmente em pós-operatório de cirurgias artroscópicas do joelho. Também se constatou que a acupuntura pode acelerar a 
recuperação do joelho operado, auxiliando no equilíbrio biodinâmico e evitando o processo inflamatório, sem a utilização de anti-inflamatórios e a volta do indivíduo operado mais rápida às suas atividades, como usa-se o joelho contralateral evitando o desconforto e diminuindo risco de infecção no joelho operado, assim demonstrando que a acupuntura é um excelente coadjuvante para o tratamento de pós-operatório em artroplastia total de joelho associado ao tratamento fisioterápico.

De acordo com estudos de Yamamura (2009), Saidah (1997) e Saidah (2001), os pontos de acupuntura mais adequados para alívio de dor no pós-operatório de joelho são: Ex-MI-1 (Heding), Ex-MI-3 (Xiyan) situados fora dos canais de energia (pontos extras); B40 (Weizhong) que se encontra no canal da Bexiga; E35 (Dubi), E36 (Zusanli) encontrados no canal do Estômago; VB34 (Yanglingquan) encontrado no canal da Vesícula Biliar; BP9 (Yinlingquan) encontrado no canal do BaçoPâncreas; R10 (Yingu) encontrado no canal dos Rins; F8 (Ququan) encontrado no canal do Fígado. Pontos estes que tem uma contribuição de grande valia para o tratamento em conjunto com a fisioterapia.

O estudo realizado por Zhang et al. (2016), utilizou os pontos E36 (Zusanli); E34 (Liangqiu); BP10 (Xuehai); BP11 (Jimen), EX-MI-1 (Heding) e EX-MI-3 (Xiyan), sendo este último estimulado com o eletroestimulador), e revelou melhora nos sintomas da osteoartrite de joelho como a dor, rigidez matinal e disfunção articular.

E em estudo realizado por Kim et al. (2017), observaram no pós-cirúrgico de LCA, com a acupuntura, melhora na dor, na queimação e sensação de calor, na rigidez em atividades diárias e esportes tendo uma significativa melhora na função da articulação do joelho. Foi realizado uma sessão na parte da manhã com o uso dos pontos BP2 (Dadu); C8 (Shaofu); E41 (Jiexi); VB41 (Zulinqi); VB43 (Jiaxi) e B66 (Zutonggu) e outra sessão na parte da tarde com o uso dos pontos B40 (Weizhong); E31 (Biguan); E33 (Yinshi); E34 (Liangqiu); BP 10 (Xuehai) e BP11 (Jimen) que foram estimulados com o eletroestimulador.

Em estudo realizado, em Israel, por Levy et al. (2018), foi relatado uma melhora de pelo menos $40 \%$ no nível médio da dor tanto em repouso quanto em movimento após acupuntura em relação ao grupo controle, em pós-operatórios.

E Siqueira et al. (2018), observou que com a utilização da acupuntura houve melhora significativa na dor de joelho em corredores, como também, melhora do equilíbrio da força muscular entre os membros evitando lesões esportivas e alterações no desempenho esportivo que permitiu a execução mais apropriada dos movimentos característicos da modalidade esportiva.

\section{Considerações Finais}

A utilização da acupuntura no tratamento de pós-operatório de LCA em jogadores de futebol apresenta muitos benefícios em conjunto com a fisioterapia, como a diminuição de infecções, melhora da biomecânica da articulação, mas principalmente ajuda no controle da dor, mostrando-se como uma ótima alternativa de técnica complementar ao tratamento, dando um conforto maior aos atletas que passaram por procedimentos cirúrgicos no joelho e acelerando o retorno às suas atividades específicas da modalidade. Entretanto, se mostra necessário mais estudos relacionados à utilização da acupuntura em pós-cirúrgico e em atletas de alto rendimento.

Com base nos artigos utilizados foi possível identificar alguns pontos principais para o tratamento de gonalgias, incluindo a lesão de LCA, sendo eles: Ex-MI-1 (Saidah, 1997; Saidah, 2001; Yamamura, 2009; Zhang et al., 2016), Ex-MI-3 (Saidah, 1997; Saidah, 2001; Yamamura, 2009; Zhang et al., 2016), B40 (Saidah, 1997; Saidah, 2001; Yamamura, 2009; Kim et al., 2017), E36 (Saidah, 1997; Saidah, 2001; Yamamura, 2009; Zhang et al., 2016), E35 (Saidah, 1997; Saidah, 2001; Yamamura, 2009), Vb34 (Saidah, 1997; Saidah, 2001, Yamamura, 2009), Bp9 (Saidah, 1997; Saidah, 2001; Yamamura, 2009), R10 (Saidah, 1997; Saidah, 2001; Yamamura, 2009), F8 (Saidah, 1997; Saidah, 2001; Yamamura, 2009), E34 (Zhang et al., 2016; Kim et al., 2017), BP10 (Zhang et al., 2016; Kim et al., 2017), BP11 (Zhang et al., 2016; Kim et al., 2017), BP2, C8, E31, E33, E41, VB41, VB43 e B66 (Kim et al., 2017). 


\section{Referências}

Arliani, G. G. et al. (2012) Lesão do ligamento cruzado anterior: tratamento e reabilitação. Perspectivas e tendências atuais. Revista Brasileira de Ortopedia, 47, $191-196$.

Arliani, G. G. et al. (2019) Tratamento das lesões do ligamento cruzado anterior em jogadores profissionais de futebol por cirurgiões ortopedistas. Revista Brasileira de Ortopedia, 54, 703-708.

Astur, D. C. et al. (2016) Lesões do ligamento cruzado anterior e do menisco no esporte: incidência, tempo de prática até a lesão e limitações causadas pelo trauma. Revista brasileira de ortopedia, 51, 652-656.

Barker, J. U. et al. (2010) Effect of graft selection on the incidence of postoperative infection in anterior cruciate ligament reconstruction. The American journal of sports medicine, 38, 281-286.

Birmingham, T. B. et al. (2008) A randomized controlled trial comparing the effectiveness of functional knee brace and neoprene sleeve use after anterior cruciate ligament reconstruction. The American journal of sports medicine, 36, 648-655.

Boden, B. P. et al. (2000) Etiology and prevention of noncontact ACL injury. The Physician and sportsmedicine, 28, 53-60.

Boone, J. Addressing the ACL. (2006) Ways to help prevent soccer's most dreaded injury. Soccer Journal, 15 , 36-7.

Capili, B, Anastasi, J. K, \& Geiger, J. N. (2010) Adverse event reporting in acupuncture clinical trials focusing on pain. The Clinical journal of pain, 26 , 43-48.

Cerulli, G. et al. (2003) In vivo anterior cruciate ligament strain behaviour during a rapid deceleration movement: case report. Knee Surgery, Sports Traumatology, Arthroscopy, 11, 307-311.

Contatore, O. A, Tesser, C. D, \& Barros, N. F. (2018) Medicina chinesa/acupuntura: apontamentos históricos sobre a colonização de um saber. História, Ciências, Saúde-Manguinhos, 25, 841-858.

Dias Junior, J. C, \& Marino, D. M. (2019) Acupuntura na prevenção de lesões musculares em atletas de futebol profissional.

Francis, A, Thomas, R. D, \& Mcgregor, A. (2001) Anterior cruciate ligament rupture: reconstruction surgery and rehabilitation: A nation-wide survey of current practice. The Knee, $8,13-18$.

Frasca, L. (2011) Desempenho na Ponta da Agulha. Rev Farmac, 44, 5.

Frobell, R. B. et al. (2010) A randomized trial of treatment for acute anterior cruciate ligament tears. New England Journal of Medicine, 363, $331-342$.

Hägglund, M. et al. (2005) Methods for epidemiological study of injuries to professional football players: developing the UEFA model. British journal of sports medicine, 39, 340-346.

Heijine, A, \& Werner, S. (2010) A 2-year follow-up of rehabilitation after ACL reconstruction using patellar tendon or hamstring tendon grafts: a prospective randomised outcome study. Knee Surgery, Sports Traumatology, Arthroscopy, 18, 805-813.

Hertel, J, Dorfman, J. H, \& Braham, R. A. (2004) Lower extremity malalignments and anterior cruciate ligament injury history. Journal of sports science \& medicine, 3, 220.

Hughes, G, \& Watkins, J. (2006) A risk-factor model for anterior cruciate ligament injury. Sports Medicine, 36, 411-428.

Jayasuriya, A. (1995) As bases científicas da acupuntura. Sohaku-in Edições.

Keays, S. L. et al. (2003) The relationship between knee strength and functional stability before and after anterior cruciate ligament reconstruction. Journal of Orthopaedic Research, 21, 231-237.

Kim, H. R. et al. (2017) Korean Medical Therapy for Knee Pain after Anterior Cruciate Ligament Reconstruction. The Acupuncture, 34, 67-79.

Lee, P. K. et al. (1975) Treatment of chronic pain with acupuncture. Jama, 232, 1133-1135.

Levy, I. et al. (2018) Postoperative analgesia by adding acupuncture to conventional therapy, a non-randomized controlled trial. Journal of Complementary and Integrative Medicine, 16, 2.

Lima, D. A. et al. (2018) Indicações cirúrgicas para reconstrução do ligamento cruzado anterior combinada com tenodese extra-articular lateral ou reconstrução do ligamento anterolateral. Revista brasileira de ortopedia, 53, 661-667.

Luna, M. P, \& Fernandes filho, J. (2005) Efeitos da Acupuntura na performance de Atletas velocista de alto rendimento do Rio de Janeiro. Fit e Perform Journal, 4, 199-214.

Machado, N. D. (2011) Vantagens da utilização da acupuntura no tratamento da osteoartrite de joelho. Escola Brasileira de Medicina Chinesa (EBRAMEC). São Paulo.

Maciocia, G. (2007) Os fundamentos da Medicina Chinesa: um texto abrangente para acupunturistas e fitoterapeutas. Ed. Rocca.

Medina, M. T. (2011) Aplicação da Acupuntura na fase pós-operatória em artroplastia total de joelho. Editor Científico, p. 41.

Min, L. S. (2004) Curso básico: Acupuntura e medicina tradicional chinesa. In: Curso básico: Acupuntura e medicina tradicional chinesa. p. $461-461$. 
Moniz, P. J. (2015) Avaliação da acupuntura no desempenho no rugby. Dissertação de Mestrado.

Musil, D, (2007) Sadovský, P, Stehlik, J. Intra-articular analgesia after anterior cruciate ligament reconstruction. Acta chirurgiae orthopaedicae et traumatologiae Cechoslovaca, 74, 182-188.

Noronha, J. C. (2006) Lesões do ligamento cruzado anterior. O Joelho. Lisboa: Lidel-edições técnicas, 1da, p. $147-82$.

Ohnishi, S. T. (2007) K.i: A key to transform the century of death to the century of life. Evidence-based Complementary and Alternative Medicine, 4, 287-92. Palastanga, N., FIeld, D., \& Soames, R. (2000) Anatomia e movimento humano: estrutura e função. (3a ed.), Ed. Manole.

Ramos, D. et al. (2019) Antonímia entre o retorno às atividades e a propriocepção efetiva da reabilitação acelerada no pós-operatório de reconstrução cirúrgica do ligamento cruzado anterior (LCA). DêCiência em Foco, 3, 82-92.

Roi, G. S. et al. (2006) Prevalence of anterior cruciate ligament reconstructions in professional soccer players. Sport sciences for health, 1, 118-121.

Rougraff, B. et al. (1993) Arthroscopic and histologic analysis of human patellar tendon autografts used for anterior cruciate ligament reconstruction. The American journal of sports medicine, 21, 277-284.

Saidah, R. et al. (1997) Tratamento das algias do joelho pela acupuntura com a utilização da técnica" AO OPOSTO" da medicina tradicional chinesa.

Saidah, R. (2001) Benefícios da acupuntura no pós-operatório das cirurgias artroscópicas no joelho. 119f. Tese (Doutorado em Medicina e Ciências Correlatas) - Faculdade de Medicina de São José do Rio Preto, São José do Rio Preto.

Saidah, R. et al. (2003) Acupuntura em relação a dor, atividade física e a necessidade de apoio para a marcha, no pós-operatório das cirurgias artroscópicas no joelho. Acta Ortopédica Brasileira, 11, 05-10.

Sanders, B. et al. (2007) Prevalence of saphenous nerve injury after autogenous hamstring harvest: an anatomic and clinical study of sartorial branch injury. Arthroscopy: The Journal of Arthroscopic \& Related Surgery, 23, 956-963.

Santos, F. A. Z. et al. (2009) Acupuntura no Sistema Único de Saúde e a inserção de profissionais não-médicos. Brazilian Journal of Physical Therapy, 13, 330334.

Santos, V. C., Kawano, M. M., \& Banja, R. A. (2008) Acupuntura na Melhora da Performance em Atletas Juvenis De Handebol. Saúde e Pesquisa, 1, 331-335.

Shelbourne, K. D. et al. (1991) Arthrofibrosis in acute anterior cruciate ligament reconstruction: the effect of timing of reconstruction and rehabilitation. The American journal of sports medicine, 19, 332-336.

Shelbourne, K. D., \& Gray, T. (1997) Anterior cruciate ligament reconstruction with autogenous patellar tendon graft followed by accelerated rehabilitation: a two-to nine-year followup. The American journal of sports medicine, 25, 786-795.

Shelbourne, K. D., \& Nitz, P. (1990) Accelerated rehabilitation after anterior cruciate ligament reconstruction. The American journal of sports medicine, 18, 292-299.

Shelbourne, K. D, Patel, D. V. (1996) Prevention of complications after autogenous bone-patellar tendon-bone ACL reconstruction. Instructional course lectures, $45,253$.

Siqueira, A. P et al. (2018) Reduction in knee pain symptoms in athletes using an acupuncture protocol. Acta ortopedica brasileira, $26,418-422$.

Siqueira, T. S., \& Viegas, S. C. (2019) Método natural de tratamento: a acupuntura no SUS de Porto Alegre. Revista Eletrônica em Gestão e Tecnologia, 5, 1424.

Soares, J. (2007) O treino do futebolista. Lesões-Nutrição. Porto Editora.

Söderman, K. et al. (2001) Injuries in adolescent female players in European football: a prospective study over one outdoor soccer season. Scandinavian journal of medicine \& science in sports, 11, 299-304.

Sun, K. et al. (2009) Anterior cruciate ligament reconstruction with bone-patellar tendon-bone autograft versus allograft. Arthroscopy: The Journal of Arthroscopic \& Related Surgery, 25, 750-759.

Tansini, S. et al. (2015) Analgesia por acupuntura versus eletroestimulação no pós-operatório de artroplastia total de joelho. Fisioterapia Ser, v. 10, n. 1, p. 4043.

Thiele, E. et al. (2009) Protocolo de reabilitação acelerada após reconstrução de ligamento cruzado anterior-dados normativos. Revista do Colégio Brasileiro de Cirurgiões, 36, 504-508.

Tyler, T. F. et al. (2004) The effect of creatine supplementation on strength recovery after anterior cruciate ligament (ACL) reconstruction: a randomized, placebo-controlled, double-blind trial. The American journal of sports medicine, 32, 383-388.

Van den berg, I. et al. (2010) Cost-effectiveness of breech version by acupuncture-type interventions on BL 67, including moxibustion, for women with a breech foetus at 33 weeks gestation: a modelling approach. Complementary therapies in medicine, 18, 67-77,

Wen, T. S. (2011) Acupuntura clássica chinesa. : Ed. Cultrix

Yamamura, I. (2009) Acupuntura Tradicional - A Arte de Inserir. Introdução LVI.

Yamamura, Y. (1993) Padronização do tratamento de algias cronicas do joelho pela medicina chinesa-acupuntura. 
Research, Society and Development, v. 10, n. 7, e24610716494, 2021

(CC BY 4.0) | ISSN 2525-3409 | DOI: http://dx.doi.org/10.33448/rsd-v10i7.16494

Yang, H. Y., Liu, T. Y., \& Gao, M. (2006) Electrical acupoint stimulation increases athletes rapid strength. Zhongguo Zhen Jiu, 26, 313-315.

Zhang, Y. et al. (2016) Influence of acupuncture in treatment of knee osteoarthritis and cartilage repairing. American journal of translational research, 8, 3995. 